\title{
Methylenetetrahydrofolate reductase tagging polymorphisms are associated with risk of esophagogastric junction adenocarcinoma: a case-control study involving 2,740 Chinese Han subjects
}

\author{
Guowen Ding ${ }^{1, *}$, Yafeng Wang ${ }^{2, *}$, Yu Chen ${ }^{3, *}$, Jun Yin ${ }^{1}$, Chao Liu1 ${ }^{1}$, Yu Fan ${ }^{4}$, Hao Qiu5 \\ Weifeng Tang ${ }^{6}$ and Shuchen Chen ${ }^{6}$ \\ ${ }^{1}$ Department of Cardiothoracic Surgery, Affiliated People's Hospital of Jiangsu University, Zhenjiang, Jiangsu Province, China \\ ${ }^{2}$ Department of Cardiology, The People's Hospital of Xishuangbanna Dai Autonomous Prefecture, Jinghong, Yunnan Province, \\ China \\ ${ }^{3}$ Department of Medical Oncology, Fujian Cancer Hospital, Fujian Medical University Cancer Hospital, Fuzhou, Fujian Province, \\ China \\ ${ }^{4}$ Department of Medical Oncology, Affiliated People's Hospital of Jiangsu University, Zhenjiang, Jiangsu Province, China \\ ${ }^{5}$ Department of Immunology, School of Medicine, Jiangsu University, Zhenjiang, Jiangsu Province, China \\ ${ }^{6}$ Department of Thoracic Surgery, Fujian Medical University Union Hospital, Fuzhou, Fujian Province, China \\ *These authors have contributed equally to this work \\ Correspondence to: Shuchen Chen, email: cscdoctor@163.com \\ Weifeng Tang, email: twf001001@126.com \\ Keywords: MTHFR; polymorphism; esophagogastric junction adenocarcinoma; risk \\ Received: May 16, $2017 \quad$ Accepted: November 13, $2017 \quad$ Published: December 01, 2017 \\ Copyright: Ding et al. This is an open-access article distributed under the terms of the Creative Commons Attribution License 3.0 \\ (CC BY 3.0), which permits unrestricted use, distribution, and reproduction in any medium, provided the original author and source \\ are credited.
}

\section{ABSTRACT}

In this study, we aimed to determine the potential association of MTHFR tagging single nucleotide polymorphisms (SNPs) with risk of developing esophagogastric junction adenocarcinoma (EGJA). MTHFR rs1801133 G>A, rs3753584 T>C, rs4845882 G $>A$, rs4846048 $A>G$ and rs9651118 $T>C$ polymorphisms were genotyped in 1,677 healthy individuals and 1,063 patients with EGJA. We found that MTHFR rs1801133 G>A polymorphism was significantly associated with the risk of developing EGJA (AA vs. GG: adjusted $P=0.001$; GA/AA vs. GG: adjusted $P=0.007$ and AA vs. GA/ GG: adjusted $P=0.001$ ). However, for MTHFR rs4845882 G $>$ A polymorphism, the decreased risk of EGJA was found in two genetic models (AA vs. GG: adjusted $P=$ 0.002 and AA vs. GA/GG: adjusted $P=0.005$ ). In addition, for MTHFR rs3753584 $\mathrm{T}>\mathrm{C}$ and rs9651118 $\mathrm{T}>\mathrm{C}$ polymorphisms, a tendency to decreased risk of EGJA was noted. In a subgroup analysis, a significantly decreased risk of EGJA in <64 years subgroup was identified. We found that MTHFR $G_{r s 1801133} T_{r s 3753584} G_{r s 4845882} A_{r s 4846048} C_{r s 9651118}$

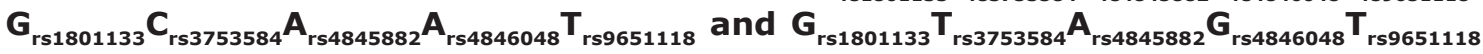
haplotypes significantly decreased the risk of EGJA $(P=0.002, P<0.001$ and $P=$ 0.038 , respectively). In conclusion, our study demonstrates that MTHFR rs 1801133 G>A may be associated with the increased risk of EGJA. Meanwhile, MTHFR rs3753584 T>C, rs4845882 G>A and rs9651118 T>C polymorphisms and haplotypes may decrease the risk of EGJA in Eastern Chinese Han population. Further studies with large sample size and detailed gene-environmental data are needed to validate our conclusion. 


\section{INTRODUCTION}

The increasing incidence of esophagogastric junction adenocarcinoma (EGJA) was observed worldwide [1-3] and was considered to have different etiology and risk factor compared with distal gastric carcinoma (GC) [4]. EGJA remains poor prognosis [5] and is a common public health problem. The vital risk factors contributing to the development of EGJA are obesity, gastroesophageal reflux disease, smoking, foods preserved by salting and low intake of fruits and vegetables et al $[6,7]$. However, these observed risk factors could not interpret the overall susceptibility to EGJA. Recently, more and more epidemiologic studies suggested that individual's genetic factor might influence the pathogenesis of EGJA.

Accumulating evidences indicate that folate insufficiency may increase the susceptibility of multiple malignancies $[8,9]$. In humans, the majority of methyl groups may be presented by folic acid for endocellular methylation reactions and DNA de novo deoxynucleoside synthesis. During DNA synthesis, lack of folate can cause uracil misincorporation and then affect the stability of DNA [10]. In folate metabolism and DNA synthesis, methylenetetrahydrofolate reductase (MTHFR) is an important enzyme which catalyzes the revivification of 5,10-methylenetetrahydrofolate to 5-methyltetrahydrofolate. And 5-methyltetrahydrofolate is a main circulating and existing form of folate and is the methyl donor for DNA methylation and remethylation procedure of homocysteine to methionine. Based on the important role of participation in both DNA synthesis and methylation, any variant of MTHFR gene may involve in the carcinogenesis.

Human MTHFR is composed of 656 amino acids. MTHFR gene is located on the short arm of Chromosome 1. The human MTHFR gene is very polymorphic (http:// www.ncbi.nlm.nih.gov/SNP) and a number of loci have been established, such as rs1537514, rs3753584, rs9651118, rs1537516, rs4845882, rs1801131, rs1801133, rs2066462, rs4846048 and rs3737967 polymorphisms, etc. Interestingly, many previous case-control studies demonstrated that MTHFR polymorphisms were correlated with the risk of multiple human malignancies [e.g., esophageal squamous cell carcinoma (ESCC) [11], gastric cardia adenocarcinoma (GCA) [12], cervicalcancer [13], breast cancer [14, 15] and childhood acute lymphoblastic leukemia [16] et al]. Thus, the single nucleotide polymorphisms (SNPs) in MTHFR genes on EGJA risk attracted our interest. Exploring the potential association of MTHFR SNPs with EGJA susceptibility may be conducive to the prevention and personalized diagnosis. In this study, we selected MTHFR tagging SNPs (rs1801133 $\mathrm{G}>\mathrm{A}, \mathrm{rs} 3753584 \mathrm{~T}>\mathrm{C}, \mathrm{rs} 4845882 \mathrm{G}>\mathrm{A}$, rs4846048 A>G and $\mathrm{rs} 9651118 \mathrm{~T}>\mathrm{C}$ ) and performed a case-control study to evaluate the effect of MTHFR genotypes for EGJA risk.

\section{RESULTS}

\section{Baseline characteristics}

A total of 1,063 sporadic patients with EGJA and 1,677 normal controls were recruited. Of the EJGA patients, 759 were male and 304 were female, with a mean age ( \pm standard deviation) of $64.19 \pm 8.63$ years. The normal controls comprised of 1,194 males and 483 females with a mean age of $63.91 \pm 10.22$ years. The demographics (age and sex) was well matched $(P=0.165$ and $P=0.909$, respectively; Table 1). Of the smoking and alcohol consumption, a significant difference was observed between EGJA patients and controls $(P<0.001$, Table 1). The frequency distribution of MTHFR genotypes was determined after genotyping the 2,740 study subjects. For MTHFR rs $1801133 \mathrm{G}>\mathrm{A}, \mathrm{rs} 3753584 \mathrm{~T}>\mathrm{C}$, rs4845882 $\mathrm{G}>\mathrm{A}$, rs4846048 $\mathrm{A}>\mathrm{G}$ and rs9651118 $\mathrm{T}>\mathrm{C}$ polymorphisms, success rates of genotyping were $99.01 \%$, $99.09 \%, 99.05 \%, 99.09 \%$ and $98.98 \%$, respectively (Table 2). In controls, the distribution of MTHFR genotype frequencies accorded with Hardy-Weinberg equilibrium (HWE), except for MTHFR rs4846048 A>G polymorphism (Table 2 ).

\section{Association of $M T H F R$ rs1801133 G $>$ A, rs3753584 T $>C$, rs4845882 $G>A$, rs4846048 A $>$ G and rs9651118 $\mathrm{T}>\mathrm{C}$ polymorphisms with EGJA}

The genotypes of MTHFR rs1801133 G>A, rs3753584 $\mathrm{T}>\mathrm{C}$, rs4845882 $\mathrm{G}>\mathrm{A}, \mathrm{rs} 4846048 \mathrm{~A}>\mathrm{G}$ and rs9651118 $\mathrm{T}>\mathrm{C}$ polymorphisms are presented in Table 3. For MTHFR rs $1801133 \mathrm{G}>\mathrm{A}$ polymorphism, the risk of developing EGJA was significant in three genetic models [AA vs. GG: crude odds ratio $(\mathrm{OR})=1.50,95 \%$ confidence interval (CI): $1.19-1.90, P=0.001 ; \mathrm{GA} /$ AA vs. GG: crude OR $=1.27,95 \%$ CI: $1.08-1.49, P=$ 0.004 and $\mathrm{AA} v s$. GA/GG: crude $\mathrm{OR}=1.45,95 \% \mathrm{CI}$ : $1.17-1.80, P=0.001$; Table 3]. Adjustment for age, sex, smoking and drinking, the similar results were also found (AA vs. GG: adjusted OR $=1.47,95 \%$ CI: $1.16-1.86$, $P=0.001 ; \mathrm{GA} / \mathrm{AA} v s . \mathrm{GG}$ : adjusted $\mathrm{OR}=1.25,95 \%$ CI: $1.06-1.47, P=0.007$ amd AA vs. GA/GG: adjusted OR $=1.43,95 \%$ CI: $1.15-1.77, P=0.001$; Table 3 ). For MTHFR rs4845882 $\mathrm{G}>\mathrm{A}$ polymorphism, the decreased risk of EGJA was found in two genetic models (AA vs. GG: crude OR $=0.47,95 \%$ CI: $0.29-0.75, P=0.002$ and AA vs. GA/GG: crude OR $=0.50,95 \%$ CI: $0.31-0.80, P$ $=0.004$; Table 3). Adjustment for age, sex, smoking and drinking, the results were not materially changed (AA vs. GG: adjusted $\mathrm{OR}=0.47,95 \% \mathrm{CI}: 0.29-0.76, P=0.002$ and AA vs. GA/GG: adjusted $\mathrm{OR}=0.50,95 \% \mathrm{CI}: 0.31-$ $0.81, P=0.005$; Table 3 ). In addition, these associations were still significant after a Bonferroni correction for multiple comparisons. 
Table 1: Distribution of selected demographic variables and risk factors in EGJA cases and controls

\begin{tabular}{|c|c|c|c|}
\hline \multirow[t]{2}{*}{ Variable } & Overall Cases $(n=1,063)$ & Overall Controls $(n=1,677)$ & $P^{\text {a }}$ \\
\hline & n (\%) & n $(\%)$ & \\
\hline Age (years) & $64.19 \pm 8.63$ & $63.91 \pm 10.22$ & 0.451 \\
\hline Age (years) & & & 0.165 \\
\hline$<64$ & $494(46.47)$ & 825 (49.19) & \\
\hline$\geq 64$ & $569(53.53)$ & $852(50.81)$ & \\
\hline Sex & & & 0.909 \\
\hline Male & $759(71.40)$ & $1194(71.20)$ & \\
\hline Female & $304(28.60)$ & $483(28.80)$ & \\
\hline Smoking status & & & $<0.001$ \\
\hline Never & $773(72.72)$ & $1323(78.89)$ & \\
\hline Ever & $290(27.28)$ & $354(21.11)$ & \\
\hline Alcohol use & & & $<0.001$ \\
\hline Never & $908(85.42)$ & $1507(89.86)$ & \\
\hline Ever & $155(14.58)$ & $170(10.14)$ & \\
\hline
\end{tabular}

a Two-sided $\chi^{2}$ test and Student $t$ test.

Table 2: Primary information for $M$ THFR polymorphisms (rs1801133 G>A, rs3753584 T>C, rs4845882 G>A, rs4846048 $\mathrm{A}>\mathrm{G}$ and $\mathrm{rs} 9651118 \mathrm{~T}>\mathrm{C}$ )

\begin{tabular}{|c|c|c|c|c|c|}
\hline Genotyped SNPs & rs1801133 G>A & rs3753584 T $>C$ & rs4845882 G>A & rs4846048 A>G & rs9651118 T $>C$ \\
\hline Chromosome & 1 & 1 & 1 & 1 & 1 \\
\hline Function & Missense & NearGene-5 & Intron & Intron & Intron \\
\hline Chr Pos (Genome Build 36.3) & 11778965 & 11787173 & 11765754 & 11768839 & 11784801 \\
\hline $\mathrm{MAF}^{\mathrm{a}}$ for Chinese in database & 0.439 & 0.093 & 0.198 & 0.105 & 0.382 \\
\hline MAF in our controls $(n=1,677)$ & 0.359 & 0.108 & 0.209 & 0.096 & 0.378 \\
\hline $\begin{array}{l}P \text { value for } \mathrm{HWE}^{\mathrm{b}} \text { test in our } \\
\text { controls }\end{array}$ & 0.679 & 0.691 & 0.972 & 0.014 & 0.270 \\
\hline Genotyping method & SNPscan & SNPscan & SNPscan & SNPscan & SNPscan \\
\hline$\%$ Genotyping value & $99.01 \%$ & $99.09 \%$ & $99.05 \%$ & $99.09 \%$ & $98.98 \%$ \\
\hline
\end{tabular}

${ }^{a}$ MAF: minor allele frequency.

${ }^{b}$ HWE: Hardy-Weinberg equilibrium.

\section{Association of MTHFR rs1801133 G $>$ A, rs3753584 $\mathrm{T}>\mathrm{C}, \mathrm{rs} 4845882 \mathrm{G}>\mathrm{A}, \mathrm{rs} 4846048 \mathrm{~A}>\mathrm{G}$ and rs9651118 $\mathrm{T}>\mathrm{C}$ polymorphisms with EGJA in Different Stratification Groups}

In the stratified analyses by sex, age, drinking and smoking, the genotype frequencies of MTHFR rs 1801133 $\mathrm{G}>\mathrm{A}$ polymorphism are listed in Table 4 . After adjustment by logistic regression analysis, the association of MTHFR rs $1801133 \mathrm{G}>\mathrm{A}$ variants with EGJA risk was evident in some subgroups [male group: AA vs. GG: adjusted OR $=1.66,95 \%$ CI $1.26-2.20, P<0.001, \mathrm{GA} / \mathrm{AA} v s . \mathrm{GG}$ : adjusted $\mathrm{OR}=1.27,95 \% \mathrm{CI} 1.05-1.53, P=0.015$ and AA vs. GA/GG: adjusted $\mathrm{OR}=1.61,95 \%$ CI 1.24-2.08, $P<0.001 ;<64$ years subgroup: AA vs. GG: adjusted OR $=1.51,95 \%$ CI $1.06-2.14, P=0.022$, GA/AA $v s$. GG: adjusted $\mathrm{OR}=1.38,95 \% \mathrm{CI} 1.09-1.74, P=0.007$ and AA vs. GA/GG: adjusted OR $=1.39,95 \%$ CI 1.00-1.92, $P=0.049 ; \geq 64$ years subgroup: AA vs. GG: adjusted OR $=1.42,95 \%$ CI 1.03-1.95, $P=0.032$ and AA vs. GA/GG: adjusted OR $=1.47,95 \%$ CI $1.10-1.96, P=0.010$; never smoking group: AA vs. GG: adjusted OR $=1.43,95 \%$ CI 1.08-1.87, $P=0.012, \mathrm{GA} / \mathrm{AA}$ vs. GG: adjusted $\mathrm{OR}=$ $1.32,95 \%$ CI $1.10-1.59, P=0.004$ and AA $v s$. GA/GG: 
Table 3: Logistic regression analyses of associations between $M T H F R$ rs1801133 G $>A$, rs3753584 $\mathrm{T}>\mathrm{C}$, rs4845882 $\mathrm{G}>\mathrm{A}, \mathrm{rs4846048} \mathrm{A}>\mathrm{G}$ and $\mathrm{rs} 9651118 \mathrm{~T}>\mathrm{C}$ polymorphisms and the risk of EGJA

\begin{tabular}{|c|c|c|c|c|c|c|c|c|}
\hline \multirow[t]{2}{*}{ Genotype } & \multicolumn{2}{|c|}{$\begin{array}{c}\text { Cases } \\
(n=1,063)\end{array}$} & \multicolumn{2}{|c|}{$\begin{array}{l}\text { Controls } \\
(n=1,677)\end{array}$} & \multirow[t]{2}{*}{$\begin{array}{c}\text { Crude OR } \\
(95 \% \mathrm{CI})\end{array}$} & \multirow[t]{2}{*}{$P$} & \multirow[t]{2}{*}{$\begin{array}{l}\text { Adjusted OR a } \\
(95 \% \mathrm{CI})\end{array}$} & \multirow[t]{2}{*}{$P$} \\
\hline & $\mathbf{n}$ & $\%$ & $\mathbf{n}$ & $\%$ & & & & \\
\hline \multicolumn{9}{|c|}{ MTHFR $\mathrm{rs} 1801133 \mathrm{G}>\mathrm{A}$} \\
\hline GG & 367 & 35.29 & 683 & 40.82 & 1.00 & & 1.00 & \\
\hline GA & 492 & 47.31 & 778 & 46.50 & $1.11(0.94-1.32)$ & 0.208 & $1.10(0.93-1.30)$ & 0.263 \\
\hline AA & 181 & 17.40 & 212 & 12.67 & $1.50(1.19-1.90)$ & 0.001 & 1.47 (1.16-1.86) & 0.001 \\
\hline $\mathrm{GA}+\mathrm{AA}$ & 673 & 64.71 & 990 & 59.18 & 1.27 (1.08-1.49) & 0.004 & $1.25(1.06-1.47)$ & 0.007 \\
\hline $\mathrm{GG}+\mathrm{GA}$ & 859 & 82.60 & 1,461 & 87.33 & 1.00 & & 1.00 & \\
\hline AA & 181 & 17.40 & 212 & 12.67 & $1.45(1.17-1.80)$ & 0.001 & $1.43(1.15-1.77)$ & 0.001 \\
\hline A allele & 854 & 41.06 & 1,202 & 35.92 & & & & \\
\hline \multicolumn{9}{|c|}{$M T H F R$ rs3753584 T>C } \\
\hline $\mathrm{TT}$ & 855 & 82.13 & 1,330 & 79.45 & 1.00 & & 1.00 & \\
\hline $\mathrm{CT}$ & 177 & 17.00 & 326 & 19.47 & $0.83(0.67-1.01)$ & 0.062 & $0.83(0.68-1.01)$ & 0.067 \\
\hline $\mathrm{CC}$ & 9 & 0.86 & 18 & 1.08 & $0.76(0.34-1.70)$ & 0.504 & $0.73(0.32-1.63)$ & 0.440 \\
\hline $\mathrm{CT}+\mathrm{CC}$ & 186 & 17.87 & 344 & 20.55 & $0.84(0.69-1.03)$ & 0.087 & $0.84(0.69-1.03)$ & 0.091 \\
\hline $\mathrm{TT}+\mathrm{CT}$ & 1032 & 99.14 & 1,656 & 98.92 & 1.00 & & 1.00 & \\
\hline $\mathrm{CC}$ & 9 & 0.86 & 18 & 1.08 & $0.80(0.36-1.79)$ & 0.591 & $0.77(0.34-1.72)$ & 0.516 \\
\hline $\mathrm{C}$ allele & 195 & 9.37 & 362 & 10.81 & & & & \\
\hline \multicolumn{9}{|c|}{ MTHFR rs4845882 G>A } \\
\hline GG & 687 & 66.06 & 1,049 & 62.66 & 1.00 & & 1.00 & \\
\hline GA & 330 & 31.73 & 552 & 32.97 & $0.89(0.75-1.05)$ & 0.153 & $0.89(0.75-1.05)$ & 0.150 \\
\hline AA & 23 & 2.21 & 73 & 4.36 & $0.47(0.29-0.75)$ & 0.002 & $0.47(0.29-0.76)$ & 0.002 \\
\hline $\mathrm{GA}+\mathrm{AA}$ & 353 & 33.94 & 625 & 37.34 & $0.86(0.73-1.01)$ & 0.074 & $0.86(0.73-1.02)$ & 0.075 \\
\hline $\mathrm{GG}+\mathrm{GA}$ & 1,017 & 97.79 & 1,601 & 95.64 & 1.00 & & 1.00 & \\
\hline AA & 23 & 2.21 & 73 & 4.36 & $0.50(0.31-0.80)$ & 0.004 & $0.50(0.31-0.81)$ & 0.005 \\
\hline A allele & 376 & 18.08 & 698 & 20.85 & & & & \\
\hline \multicolumn{9}{|c|}{$M T H F R$ rs4846048 A >G } \\
\hline AA & 860 & 82.61 & 1,378 & 82.32 & 1.00 & & 1.00 & \\
\hline $\mathrm{AG}$ & 171 & 16.43 & 272 & 16.25 & $0.98(0.80-1.21)$ & 0.883 & $0.99(0.80-1.22)$ & 0.921 \\
\hline GG & 10 & 0.96 & 24 & 1.43 & $0.65(0.31-1.37)$ & 0.260 & $0.63(0.30-1.34)$ & 0.230 \\
\hline $\mathrm{AG}+\mathrm{GG}$ & 181 & 17.39 & 296 & 17.68 & $0.98(0.80-1.20)$ & 0.845 & $0.98(0.80-1.21)$ & 0.870 \\
\hline $\mathrm{AA}+\mathrm{AG}$ & 1,031 & 99.04 & 1,650 & 98.57 & 1.00 & & 1.00 & \\
\hline GG & 10 & 0.96 & 24 & 1.43 & $0.67(0.32-1.40)$ & 0.284 & $0.65(0.31-1.36)$ & 0.250 \\
\hline G allele & 191 & 9.17 & 320 & 9.56 & & & & \\
\hline \multicolumn{9}{|c|}{ MTHFR rs9651118 T>C } \\
\hline TT & 423 & 40.75 & 638 & 38.11 & 1.00 & & 1.00 & \\
\hline $\mathrm{TC}$ & 486 & 46.82 & 808 & 48.27 & $0.86(0.73-1.02)$ & 0.075 & $0.87(0.74-1.03)$ & 0.105 \\
\hline
\end{tabular}

(Continued) 


\begin{tabular}{|c|c|c|c|c|c|c|c|c|}
\hline \multirow[t]{2}{*}{ Genotype } & \multicolumn{2}{|c|}{$\begin{array}{c}\text { Cases } \\
(n=1,063)\end{array}$} & \multicolumn{2}{|c|}{$\begin{array}{l}\text { Controls } \\
(n=1,677)\end{array}$} & \multirow[t]{2}{*}{$\begin{array}{c}\text { Crude OR } \\
(95 \% \mathrm{CI})\end{array}$} & \multirow[t]{2}{*}{$P$} & \multirow[t]{2}{*}{$\begin{array}{l}\text { Adjusted OR }{ }^{\mathrm{a}} \\
\quad(95 \% \mathrm{CI})\end{array}$} & \multirow[t]{2}{*}{$P$} \\
\hline & $\mathrm{n}$ & $\%$ & n & $\%$ & & & & \\
\hline $\mathrm{CC}$ & 129 & 12.43 & 228 & 13.62 & $0.81(0.63-1.04)$ & 0.094 & $0.83(0.65-1.07)$ & 0.150 \\
\hline $\mathrm{TC}+\mathrm{CC}$ & 615 & 59.25 & 1,036 & 61.89 & $0.90(0.76-1.05)$ & 0.171 & $0.91(0.78-1.07)$ & 0.260 \\
\hline $\mathrm{TT}+\mathrm{TC}$ & 909 & 87.57 & 1,446 & 86.38 & 1.00 & & 1.00 & \\
\hline $\mathrm{CC}$ & 129 & 12.43 & 228 & 13.62 & $0.90(0.71-1.13)$ & 0.372 & $0.92(0.73-1.16)$ & 0.489 \\
\hline $\mathrm{C}$ allele & 744 & 35.84 & 1,264 & 37.75 & & & & \\
\hline
\end{tabular}

${ }^{a}$ Adjusted for age, sex, smoking and drinking status; Bold values are statistically significant $(P<0.05)$.

Table 4: Stratified analyses between MTHFR rs1801133 G>A polymorphism and EGJA risk by sex, age, smoking status and alcohol consumption

\begin{tabular}{|c|c|c|c|c|c|c|c|c|}
\hline \multirow[t]{2}{*}{ Variable } & \multicolumn{3}{|c|}{$\begin{array}{c}\text { MTHFR rs1801133 G>A } \\
\text { (case/control) }^{\mathrm{a}}\end{array}$} & \multicolumn{3}{|c|}{ Adjusted OR ${ }^{\mathrm{b}}(95 \% \mathrm{CI}) ; P$} & \multirow[b]{2}{*}{$\mathbf{G A} / \mathbf{A A}$} & \multirow[b]{2}{*}{ AA vs. (GA/GG) } \\
\hline & GG & GA & $\mathbf{A A}$ & GG & GA & $\mathbf{A A}$ & & \\
\hline \multicolumn{9}{|l|}{ Sex } \\
\hline Male & $260 / 485$ & $350 / 563$ & $135 / 142$ & 1.00 & $\begin{array}{c}1.10(0.90-1.34) ; \\
P: 0.360\end{array}$ & $\begin{array}{c}1.66(1.26-2.20) \\
\quad P:<0.001\end{array}$ & $\begin{array}{c}1.27(1.05-1.53) \\
P: 0.015\end{array}$ & $\begin{array}{c}1.61(1.24-2.08) \\
P:<0.001\end{array}$ \\
\hline Female & $107 / 198$ & $142 / 215$ & $46 / 70$ & 1.00 & $\begin{array}{c}1.10(0.80-1.50) ; \\
P: 0.575\end{array}$ & $\begin{array}{c}1.03(0.66-1.60) \\
P: 0.909\end{array}$ & $\begin{array}{c}1.17(0.86-1.59) ; \\
P: 0.311\end{array}$ & $\begin{array}{c}1.01(0.67-1.53) ; \\
P: 0.954\end{array}$ \\
\hline \multicolumn{9}{|l|}{ Age } \\
\hline$<64$ & $173 / 362$ & $233 / 363$ & $76 / 98$ & 1.00 & $\begin{array}{c}1.24(0.97-1.58) \text {; } \\
P: 0.089\end{array}$ & $\begin{array}{c}1.51(1.06-2.14) \\
P: 0.022\end{array}$ & $\begin{array}{c}1.38(1.09-1.74) \\
P: 0.007\end{array}$ & $\begin{array}{c}1.39(1.00-1.92) \\
P: 0.049\end{array}$ \\
\hline$\geq 64$ & $194 / 321$ & $259 / 415$ & $105 / 114$ & 1.00 & $\begin{array}{c}0.97(0.77-1.23) ; \\
P: 0.822\end{array}$ & $\begin{array}{l}1.42(1.03-1.95) \\
\quad P: 0.032\end{array}$ & $\begin{array}{c}1.12(0.90-1.40) \\
\quad P: 0.312\end{array}$ & $\begin{array}{c}1.47(1.10-1.96) \\
P: 0.010\end{array}$ \\
\hline \multicolumn{9}{|l|}{$\begin{array}{l}\text { Smoking } \\
\text { status }\end{array}$} \\
\hline Never & $263 / 549$ & $368 / 603$ & $123 / 168$ & 1.00 & $\begin{array}{c}1.19(0.98-1.44) ; \\
P: 0.085\end{array}$ & $\begin{array}{c}1.43(1.08-1.87) \\
\quad P: 0.012\end{array}$ & $\begin{array}{c}1.32(1.10-1.59) \\
P: 0.004\end{array}$ & $\begin{array}{c}1.33(1.04-1.72) \\
P: 0.026\end{array}$ \\
\hline Ever & $104 / 134$ & $124 / 175$ & $58 / 44$ & 1.00 & $\begin{array}{c}0.86(0.61-1.22) \\
\quad P: 0.396\end{array}$ & $\begin{array}{c}1.62(1.01-2.61) \\
\quad P: 0.046\end{array}$ & $\begin{array}{c}1.04(0.75-1.45) \\
P: 0.805\end{array}$ & $\begin{array}{c}1.79(1.15-2.76) \\
P: 0.009\end{array}$ \\
\hline \multicolumn{9}{|l|}{$\begin{array}{l}\text { Alcohol } \\
\text { consumption }\end{array}$} \\
\hline Never & $308 / 631$ & $426 / 686$ & $152 / 187$ & 1.00 & $\begin{array}{c}1.19(0.99-1.42) ; \\
P: 0.065\end{array}$ & $\begin{array}{c}1.54(1.20-1.98) \\
P: 0.001\end{array}$ & $\begin{array}{c}1.35(1.13-1.60) \\
P: 0.001\end{array}$ & $\begin{array}{c}1.45(1.15-1.82) \\
P: 0.002\end{array}$ \\
\hline Ever & $59 / 52$ & $66 / 92$ & $29 / 25$ & 1.00 & $\begin{array}{c}0.60(0.36-1.00) \\
P: 0.051\end{array}$ & $\begin{array}{c}1.10(0.56-2.19) \\
P: 0.780\end{array}$ & $\begin{array}{c}0.70(0.43-1.13) \\
P: 0.138\end{array}$ & $\begin{array}{c}1.47(0.80-2.73) ; \\
P: 0.217\end{array}$ \\
\hline
\end{tabular}

${ }^{a}$ The genotyping was successful in 1063 (97.84\%) EGJA cases, and 1677 (99.76\%) controls for MTHFR rs1801133 G>A;

${ }^{\mathrm{b}}$ Adjusted for age, sex, smoking status and alcohol consumption (besides stratified factors accordingly) in a logistic regression model;

adjusted $\mathrm{OR}=1.33,95 \%$ CI $1.04-1.72, P=0.026$; ever smoking group: AA vs. GG: adjusted $\mathrm{OR}=1.62,95 \% \mathrm{CI}$ $1.01-2.61, P=0.046$ and AA $v s$. GA/GG: adjusted OR $=1.79,95 \%$ CI $1.15-2.76, P=0.009$ and never drinking group: AA vs. GG: adjusted OR $=1.54,95 \%$ CI $1.20-1.98$,
$P=0.001, \mathrm{GA} / \mathrm{AA} v s . \mathrm{GG}:$ adjusted $\mathrm{OR}=1.35,95 \% \mathrm{CI}$ $1.13-1.60, P=0.001$ and $\mathrm{AA}$ vs. GA/GG: adjusted $\mathrm{OR}=$ $1.45,95 \%$ CI $1.15-1.82, P=0.002$; Table 4$)]$.

Table 5 summarizes the results of association between MTHFR rs3753584 $\mathrm{T}>\mathrm{C}$ polymorphism and 
Table 5: Stratified analyses between MTHFR rs3753584 T>C polymorphism and EGJA risk by sex, age, smoking status and alcohol consumption

\begin{tabular}{|c|c|c|c|c|c|c|c|c|}
\hline \multirow[t]{2}{*}{ Variable } & \multicolumn{3}{|c|}{$\begin{array}{l}\text { MTHFR rs3753584 } \\
\mathrm{T}>\mathrm{C} \text { (case/control) }^{\mathrm{a}}\end{array}$} & \multicolumn{3}{|c|}{ Adjusted OR ${ }^{\mathrm{b}}(95 \% \mathrm{CI}) ; P$} & \multirow[b]{2}{*}{ TC / CC } & \multirow[b]{2}{*}{ CC vs. (TC/TT) } \\
\hline & TT & $\mathbf{T C}$ & $\mathbf{C C}$ & TT & TC & $\mathrm{CC}$ & & \\
\hline \multicolumn{9}{|l|}{ Sex } \\
\hline Male & $613 / 950$ & $126 / 226$ & $7 / 15$ & 1.00 & $\begin{array}{c}0.85(0.67-1.08) ; \\
P: 0.177\end{array}$ & $\begin{array}{c}0.69(0.28-1.70) ; \\
P: 0.415\end{array}$ & $\begin{array}{c}0.85(0.67-1.08) \\
P: 0.184\end{array}$ & $\begin{array}{c}0.72(0.29-1.78) ; \\
P: 0.471\end{array}$ \\
\hline Female & $242 / 380$ & $51 / 100$ & $2 / 3$ & 1.00 & $\begin{array}{c}0.80(0.55-1.17) ; \\
P: 0.252\end{array}$ & $\begin{array}{c}0.61(0.08-4.43) ; \\
P: 0.622\end{array}$ & $\begin{array}{c}0.83(0.57-1.20) \\
P: 0.319\end{array}$ & $\begin{array}{c}0.64(0.09-4.72) ; \\
P: 0.663\end{array}$ \\
\hline \multicolumn{9}{|l|}{ Age } \\
\hline$<64$ & $398 / 640$ & $79 / 177$ & $5 / 7$ & 1.00 & $\begin{array}{c}0.70(0.52-0.93) \\
P: 0.016\end{array}$ & $\begin{array}{c}0.99(0.31-3.19) ; \\
P: 0.987\end{array}$ & $\begin{array}{c}0.73(0.55-0.97) \\
P: 0.032\end{array}$ & $\begin{array}{c}1.08(0.34-3.47) ; \\
P: 0.899\end{array}$ \\
\hline$\geq 64$ & $457 / 691$ & $98 / 149$ & $4 / 11$ & 1.00 & $\begin{array}{c}0.98(0.74-1.30) ; \\
P: 0.881\end{array}$ & $\begin{array}{c}0.53(0.17-1.68) ; \\
P: 0.282\end{array}$ & $\begin{array}{c}0.97(0.73-1.28) \\
P: 0.813\end{array}$ & $\begin{array}{c}0.54(0.17-1.71) ; \\
P: 0.296\end{array}$ \\
\hline \multicolumn{9}{|l|}{$\begin{array}{l}\text { Smoking } \\
\text { status }\end{array}$} \\
\hline Never & $619 / 1,058$ & $131 / 249$ & $4 / 14$ & 1.00 & $\begin{array}{c}0.88(0.70-1.11) \\
P: 0.283\end{array}$ & $\begin{array}{c}0.47(0.16-1.45) ; \\
P: 0.189\end{array}$ & $\begin{array}{c}0.88(0.70-1.11) \\
P: 0.288\end{array}$ & $\begin{array}{c}0.49(0.16-1.51) ; \\
P: 0.216\end{array}$ \\
\hline Ever & $236 / 272$ & $46 / 77$ & $5 / 4$ & 1.00 & $\begin{array}{c}0.74(0.49-1.11) \\
P: 0.144\end{array}$ & $\begin{array}{c}1.22(0.31-4.74) ; \\
P: 0.776\end{array}$ & $\begin{array}{c}0.77(0.52-1.15) \\
P: 0.202\end{array}$ & $\begin{array}{c}1.29(0.33-5.04) ; \\
P: 0.711\end{array}$ \\
\hline \multicolumn{9}{|c|}{$\begin{array}{l}\text { Alcohol } \\
\text { consumption }\end{array}$} \\
\hline Never & $729 / 1,190$ & $152 / 299$ & $6 / 16$ & 1.00 & $\begin{array}{c}0.81(0.65-1.00) ; \\
P: 0.052\end{array}$ & $\begin{array}{c}0.59(0.23-1.52) ; \\
P: 0.273\end{array}$ & $\begin{array}{c}0.82(0.66-1.01) \\
P: 0.064\end{array}$ & $\begin{array}{c}0.63(0.24-1.61) ; \\
P: 0.331\end{array}$ \\
\hline Ever & $126 / 140$ & $25 / 27$ & $3 / 2$ & 1.00 & $\begin{array}{c}1.11(0.60-2.06) ; \\
P: 0.748\end{array}$ & $\begin{array}{c}1.52(0.24-9.63) \\
P: 0.657\end{array}$ & $\begin{array}{c}1.14(0.63-2.07) \\
P: 0.668\end{array}$ & $\begin{array}{c}1.49(0.24-9.44) \\
P: 0.669\end{array}$ \\
\hline
\end{tabular}

a The genotyping was successful in 1063 (97.93\%) EGJA cases, and 1677 (99.82\%) controls for MTHFR rs3753584 T>C; ${ }^{\mathrm{b}}$ Adjusted for age, sex, smoking status and alcohol consumption (besides stratified factors accordingly) in a logistic regression model;

EGJA risk in the stratified analysis. We found that MTHFR rs3753584 $\mathrm{T}>\mathrm{C}$ polymorphism was associated with the decreased risk of EGJA in $<64$ years subgroup [TC vs. TT: adjusted $\mathrm{OR}=0.70,95 \%$ CI $0.52-0.93, P=0.016$ and TC/CC vs. TT: adjusted OR $=0.73,95 \%$ CI $0.55-0.97, P$ $=0.032($ Table 5$)]$.

The results of association between MTHFR rs4845882 $\mathrm{G}>\mathrm{A}$ polymorphism and EGJA risk in the stratified analyses are summarized in Table 6 . We found that MTHFR rs4845882 $\mathrm{G}>\mathrm{A}$ polymorphism decreased the risk of EGJA in several subgroups [male group: AA vs. GG: adjusted $\mathrm{OR}=0.47,95 \%$ CI $0.27-0.83, P=$ 0.009 and AA vs. GA/GG: adjusted $\mathrm{OR}=0.50,95 \% \mathrm{CI}$ $0.29-0.87, P=0.014 ;<64$ years subgroup: AA $v s . \mathrm{GG}$ : adjusted $\mathrm{OR}=0.41,95 \% \mathrm{CI} 0.20-0.84, P=0.015$ and AA vs. GA/GG: adjusted OR $=0.45,95 \%$ CI $0.22-0.91$, $P=0.027$; never smoking group: AA vs. GG: adjusted $\mathrm{OR}=0.37,95 \%$ CI $0.21-0.67, P=0.001$ and AA $v s$.
GA/GG: adjusted OR $=0.39,95 \%$ CI $0.22-0.70, P=$ 0.002 and never drinking group: AA vs. GG: adjusted OR $=0.44,95 \%$ CI $0.26-0.74, P=0.002$, GA/AA vs. GG: adjusted $\mathrm{OR}=0.83,95 \% \mathrm{CI} 0.69-0.98, P=0.032$ and AA $v s$. GA/GG: adjusted $\mathrm{OR}=0.48,95 \%$ CI $0.29-0.80$, $P=0.005$ (Table 6)].

Table 7 lists MTHFR rs4846048 A $>\mathrm{G}$ genotype frequencies in the stratified analysis. We found no significant difference in genotype distribution of MTHFR rs4846048 A $>$ G polymorphism among EGJA cases and non-cancer controls.

The results of relationship between MTHFR rs9651118 $\mathrm{T}>\mathrm{C}$ polymorphism and EGJA risk in the stratified analyses are summarized in Table 8. We found that MTHFR rs9651118 T $>$ C polymorphism was associated with the decreased risk of EGJA in $<64$ years subgroup [TC vs. TT: adjusted OR $=0.78,95 \%$ CI $0.61-$ $0.99, P=0.040$ (Table 8)]. 
Table 6: Stratified analyses between $M T H F R$ rs4845882 G>A polymorphism and EGJA risk by sex, age, smoking status and alcohol consumption

\begin{tabular}{|c|c|c|c|c|c|c|c|c|}
\hline \multirow[t]{2}{*}{ Variable } & \multicolumn{3}{|c|}{$\begin{array}{l}M T H F R \text { rs } 4845882 \\
\text { G }>\text { A } \text { (case/control) }^{\mathrm{a}} \\
\end{array}$} & \multicolumn{3}{|c|}{ Adjusted OR ${ }^{\mathrm{b}}(95 \% \mathrm{CI}) ; P$} & \multirow[b]{2}{*}{ GA /AA } & \multirow[b]{2}{*}{ AA vs. (GA/GG) } \\
\hline & GG & GA & $\mathbf{A A}$ & GG & GA & $\mathbf{A A}$ & & \\
\hline \multicolumn{9}{|l|}{ Sex } \\
\hline Male & $492 / 746$ & $237 / 391$ & $17 / 54$ & 1.00 & $\begin{array}{c}0.89(0.73-1.09) \\
P: 0.268\end{array}$ & $\begin{array}{c}0.47(0.27-0.83) \\
P: 0.009\end{array}$ & $\begin{array}{c}0.86(0.71-1.05) \\
P: 0.113\end{array}$ & $\begin{array}{c}0.50(0.29-0.87) \\
P: 0.014\end{array}$ \\
\hline Female & $195 / 303$ & $93 / 161$ & $6 / 19$ & 1.00 & $\begin{array}{c}0.86(0.63-1.18) \\
P: 0.354\end{array}$ & $\begin{array}{c}0.43(0.16-1.12) \\
P: 0.084\end{array}$ & $\begin{array}{c}0.86(0.63-1.17) \\
P: 0.330\end{array}$ & $\begin{array}{c}0.46(0.18-1.21) \\
P: 0.117\end{array}$ \\
\hline \multicolumn{9}{|l|}{ Age } \\
\hline$<64$ & $320 / 507$ & $152 / 279$ & $10 / 37$ & 1.00 & $\begin{array}{c}0.83(0.65-1.06) \\
P: 0.132\end{array}$ & $\begin{array}{c}0.41(0.20-0.84) \\
P: 0.015\end{array}$ & $\begin{array}{c}0.81(0.64-1.02) \\
P: 0.077\end{array}$ & $\begin{array}{c}0.45(0.22-0.91) \\
P: 0.027\end{array}$ \\
\hline$\geq 64$ & $367 / 542$ & $178 / 273$ & $13 / 36$ & 1.00 & $\begin{array}{c}0.94(0.74-1.18) \\
P: 0.566\end{array}$ & $\begin{array}{c}0.53(0.28-1.01) \\
P: 0.052\end{array}$ & $\begin{array}{c}0.91(0.73-1.14) \\
P: 0.424\end{array}$ & $\begin{array}{c}0.55(0.29-1.04) \\
P: 0.068\end{array}$ \\
\hline \multicolumn{9}{|l|}{$\begin{array}{l}\text { Smoking } \\
\text { status }\end{array}$} \\
\hline Never & $496 / 832$ & $243 / 427$ & $14 / 62$ & 1.00 & $\begin{array}{c}0.92(0.76-1.12) ; \\
P: 0.416\end{array}$ & $\begin{array}{c}0.37(0.21-0.67) \\
P: 0.001\end{array}$ & $\begin{array}{c}0.89(0.73-1.07) \\
P: 0.207\end{array}$ & $\begin{array}{c}0.39(0.22-0.70) \\
P: 0.002\end{array}$ \\
\hline Ever & $191 / 217$ & $87 / 125$ & $9 / 11$ & 1.00 & $\begin{array}{c}0.81(0.58-1.14) \\
P: 0.220\end{array}$ & $\begin{array}{c}0.96(0.38-2.40) \\
P: 0.927\end{array}$ & $\begin{array}{c}0.83(0.60-1.16) \\
P: 0.269\end{array}$ & $\begin{array}{c}1.04(0.42-2.59) ; \\
P: 0.939\end{array}$ \\
\hline \multicolumn{9}{|c|}{$\begin{array}{l}\text { Alcohol } \\
\text { consumption }\end{array}$} \\
\hline Never & $591 / 938$ & $276 / 501$ & $19 / 66$ & 1.00 & $\begin{array}{c}0.85(0.71-1.01) \\
P: 0.065\end{array}$ & $\begin{array}{c}0.44(0.26-0.74) \\
P: 0.002\end{array}$ & $\begin{array}{c}0.83(0.69-0.98) \\
P: 0.032\end{array}$ & $\begin{array}{c}0.48(0.29-0.80) \\
P: 0.005\end{array}$ \\
\hline Ever & $96 / 111$ & $54 / 51$ & $4 / 7$ & 1.00 & $\begin{array}{c}1.30(0.80-2.12) \\
P: 0.293\end{array}$ & $\begin{array}{c}0.85(0.23-3.19) ; \\
P: 0.813\end{array}$ & $\begin{array}{c}1.25(0.78-2.02) \\
P: 0.355\end{array}$ & $\begin{array}{c}0.78(0.21-2.87) ; \\
P: 0.708\end{array}$ \\
\hline
\end{tabular}

a The genotyping was successful in 1063 (97.84\%) EGJA cases, and 1677 (99.82\%) controls for MTHFR rs4845882 G>A; ${ }^{\mathrm{b}}$ Adjusted for age, sex, smoking status and alcohol consumption (besides stratified factors accordingly) in a logistic regression model;

\section{SNP haplotypes}

We used a SHESIS software (http://analysis.bio-X. $\mathrm{cn} /$ myAnalysis.php) [17] to construct haplotypes of MTHFR gene (Table 9). Finally, five MTHFR haplotypes were identified. When MTHFR A $\mathrm{A}_{\mathrm{rs} 1801133} \mathrm{~T}_{\mathrm{rs} 3753584} \mathrm{G}_{\mathrm{rs} 4845882} \mathrm{~A}$ ${ }_{\mathrm{rs} 4846048} \mathrm{~T}_{\mathrm{rs} 9651118}$ haplotype was used as reference, we found that MTHFR $\mathrm{G}_{\mathrm{rs} 1801133} \mathrm{~T}_{\mathrm{rs} 3753584} \mathrm{G}_{\mathrm{rs} 4845882} \mathrm{~A}_{\mathrm{rs} 4846048} \mathrm{C}_{\mathrm{rs} 9651118}, \mathrm{G}_{\mathrm{rs} 1}$ ${ }_{801133} \mathrm{C}_{\mathrm{rs} 3753584} \mathrm{~A}_{\mathrm{rs} 4845882} \mathrm{~A}_{\mathrm{rs} 4846048} \mathrm{~T}_{\mathrm{rs} 9651118}$ and $\mathrm{G}_{\mathrm{rs} 1801133} \mathrm{~T}_{\mathrm{rs} 3753584} \mathrm{~A}$ ${ }_{\mathrm{rs} 4845882} \mathrm{G}_{\mathrm{rs} 4846048} \mathrm{~T}_{\mathrm{rs} 9651118}$ haplotypes significantly decreased the risk of EGJA $(P=0.002, P<0.001$ and $P=0.038$, respectively, Table 9).

\section{DISCUSSION}

Incidence of EGJA has increased over the past two decades [18, 19]. Many studies demonstrated that the morbidity of EGJA was increased in Asian countries, such as China, Korea and Japan [19-21]. However, the etiology of EGJA remains unknown. In this study, we explored the association between MTHFR rs1801133 G>A, rs3753584 $\mathrm{T}>\mathrm{C}, \mathrm{rs} 4845882 \mathrm{G}>\mathrm{A}$, rs4846048 $\mathrm{A}>\mathrm{G}$ and $\mathrm{rs} 9651118$ $\mathrm{T}>\mathrm{C}$ polymorphisms and EGJA risk in Eastern Chinese Han population. We found that MTHFR rs1801133 G>A might be associated with the increased risk of EGJA. Meanwhile, MTHFR rs3753584 T $>$ C, rs4845882 G $>$ A and rs9651118 $\mathrm{T}>\mathrm{C}$ polymorphisms decreased the risk of EGJA.

MTHFR gene lies in 1p36.3 and contains 11 exons with a length of about $1980 \mathrm{bp}$. In exon 4, a G to A variant at nucleotide 677 locus (rs1801133 G>A) directly leads to valine substitution for alanine, which is relevant to a reduction of MTHFR activity [22]. The individuals who carry heterozygous genetype (GA genetype) of MTHFR rs1801133 G>A polymorphism have $70 \%$ of normal enzyme activity, however, those who carry homozygous 
Table 7: Stratified analyses between $M T H F R$ rs4846048 A>G polymorphism and EGJA risk by sex, age, smoking status and alcohol consumption

\begin{tabular}{|c|c|c|c|c|c|c|c|c|}
\hline \multirow[t]{2}{*}{ Variable } & \multicolumn{3}{|c|}{$\begin{array}{l}M T H F R \text { rs } 4846048 \\
A>G(\text { case/control) }\end{array}$} & \multicolumn{3}{|c|}{ Adjusted OR ${ }^{\mathrm{b}}(95 \% \mathrm{CI}) ; P$} & \multirow[b]{2}{*}{ AG/GG } & \multirow[b]{2}{*}{ GG vs. (AG/AA) } \\
\hline & $\mathbf{A A}$ & $\mathbf{A G}$ & GG & $\mathbf{A A}$ & AG & GG & & \\
\hline \multicolumn{9}{|l|}{ Sex } \\
\hline Male & $615 / 984$ & $124 / 189$ & $7 / 18$ & 1.00 & $\begin{array}{c}1.04(0.81-1.33) \\
P: 0.772\end{array}$ & $\begin{array}{c}0.58(0.24-1.40) \\
P: 0.227\end{array}$ & $\begin{array}{c}1.02(0.80-1.29) \\
P: 0.904\end{array}$ & $\begin{array}{c}0.58(0.24-1.41) ; \\
P: 0.233\end{array}$ \\
\hline Female & $245 / 394$ & $47 / 83$ & $3 / 6$ & 1.00 & $\begin{array}{c}0.88(0.59-1.30) \\
P: 0.522\end{array}$ & $\begin{array}{c}0.73(0.18-2.96) ; \\
P: 0.659\end{array}$ & $\begin{array}{c}0.90(0.61-1.32) \\
P: 0.597\end{array}$ & $\begin{array}{c}0.77(0.19-3.11) ; \\
P: 0.711\end{array}$ \\
\hline \multicolumn{9}{|l|}{ Age } \\
\hline$<64$ & $398 / 677$ & $78 / 134$ & $6 / 12$ & 1.00 & $\begin{array}{c}0.99(0.73-1.34) \\
P: 0.927\end{array}$ & $\begin{array}{c}0.74(0.27-2.02) \\
P: 0.562\end{array}$ & $\begin{array}{c}0.99(0.74-1.34) \\
P: 0.955\end{array}$ & $\begin{array}{c}0.76(0.28-2.07) ; \\
P: 0.590\end{array}$ \\
\hline$\geq 64$ & $462 / 701$ & $93 / 138$ & $4 / 12$ & 1.00 & $\begin{array}{c}1.00(0.75-1.33) \\
P: 0.981\end{array}$ & $\begin{array}{c}0.48(0.15-1.51) \\
P: 0.210\end{array}$ & $\begin{array}{c}0.97(0.74-1.29) \\
P: 0.855\end{array}$ & $\begin{array}{c}0.49(0.16-1.53) ; \\
P: 0.221\end{array}$ \\
\hline \multicolumn{9}{|l|}{$\begin{array}{l}\text { Smoking } \\
\text { status }\end{array}$} \\
\hline Never & $624 / 1,081$ & $125 / 220$ & $5 / 20$ & 1.00 & $\begin{array}{c}0.96(0.75-1.22) \\
P: 0.725\end{array}$ & $\begin{array}{c}0.43(0.16-1.16) \\
P: 0.095\end{array}$ & $\begin{array}{c}0.94(0.74-1.19) \\
P: 0.611\end{array}$ & $\begin{array}{c}0.45 \text { (0.17-1.19); } \\
P: 0.107\end{array}$ \\
\hline Ever & $236 / 297$ & $46 / 52$ & $5 / 4$ & 1.00 & $\begin{array}{c}1.13(0.73-1.75) \\
P: 0.582\end{array}$ & $\begin{array}{c}1.49(0.39-5.72) ; \\
P: 0.563\end{array}$ & $\begin{array}{c}1.17(0.77-1.78) ; \\
P: 0.473\end{array}$ & $\begin{array}{c}1.47(0.38-5.66) ; \\
P: 0.572\end{array}$ \\
\hline \multicolumn{9}{|c|}{$\begin{array}{l}\text { Alcohol } \\
\text { consumption }\end{array}$} \\
\hline Never & $736 / 1,236$ & $145 / 248$ & $6 / 21$ & 1.00 & $\begin{array}{c}0.96(0.77-1.20) \\
P: 0.709\end{array}$ & $\begin{array}{c}0.47(0.19-1.16) ; \\
P: 0.101\end{array}$ & $\begin{array}{c}0.95(0.76-1.18) \\
P: 0.613\end{array}$ & $\begin{array}{c}0.48(0.19-1.19) ; \\
P: 0.115\end{array}$ \\
\hline Ever & $124 / 142$ & $26 / 24$ & $4 / 3$ & 1.00 & $\begin{array}{c}1.31(0.70-2.46) \\
P: 0.406\end{array}$ & $\begin{array}{c}1.77(0.36-8.68) ; \\
P: 0.483\end{array}$ & $\begin{array}{c}1.35(0.74-2.46) ; \\
P: 0.323\end{array}$ & $\begin{array}{c}1.70(0.35-8.32) ; \\
P: 0.512\end{array}$ \\
\hline
\end{tabular}

a The genotyping was successful in 1063 (97.93\%) EGJA cases, and 1677 (99.82\%) controls for MTHFR rs4846048 A>G; ${ }^{\mathrm{b}}$ Adjusted for age, sex, smoking status and alcohol consumption (besides stratified factors accordingly) in a logistic regression model;

genotype (AA genetype) have only $30 \%$ of normal enzyme activity [23]. A case-control study reported that rs1801133 AA genotype was associated an increased risk of GCA [24]. Another case-control study also found that MTHFR rs1801133 AA and GA genotypes were associated the increased risk of GCA [25]. These results were in accordance with our conclusions. In the future, more replicated study should be conducted to verify these primary findings.

MTHFR rs3753584 $\mathrm{T}>\mathrm{C}$ is situated in the intron region of $M T H F R$ gene. There were only a few studies focusing on the association between MTHFR rs3753584 $\mathrm{T}>\mathrm{C}$ and cancer risk. A previous study found that there was an increased lung cancer risk in carriers of MTHFR rs3753584 CC genotype compared with carriers of rs3753584 TT genotype [26]. However, no association was found between ESCC risk and MTHFR rs3753584 T>C polymorphism [11]. In addition, Wang et al. also reported that MTHFR rs3753584 T>C was not associated with GCA risk [12]. The present study concluded that rs3753584 TC and $\mathrm{TC} / \mathrm{CC}$ genotypes were related to a decreased EGJA risk in $<64$ years subgroup. These apparent discrepancy findings may be due to the insufficient sample size. In the future, more studies with large sample size and detailed environmental factors are indispensable to explore the relationship between $M T H F R$ rs3753584 T $>C$ and the risk of different cancers.

MTHFR rs4845882 G>A polymorphism lies in a intron region and is almost complete linkage disequilibrium (LD) with MTHFR rs1801131 A>C locus. Shen et al. found there was no significant relationship between MTHFR rs4845882 G>A polymorphism and gastric cancer risk [27]. Additionally, the association between MTHFR rs4845882 G>A and GCA risk was not concluded in a recent sutdy [12]. However, our study saw a decreased EGJA risk in the individuals carrying 
Table 8: Stratified analyses between MTHFR rs9651118 T>C polymorphism and EGJA risk by sex, age, smoking status and alcohol consumption

\begin{tabular}{|c|c|c|c|c|c|c|c|c|}
\hline \multirow[t]{2}{*}{ Variable } & \multicolumn{3}{|c|}{$\begin{array}{c}\text { MTHFR } \operatorname{rs9651118} \mathrm{T}>\mathrm{C} \\
\left(_{(\text {case } / \text { control) }}\right.\end{array}$} & \multicolumn{3}{|c|}{ Adjusted OR ${ }^{\mathrm{b}}(95 \%$ CI $) ; P$} & \multirow[b]{2}{*}{$\mathrm{TC} / \mathrm{CC}$} & \multirow[b]{2}{*}{ CC vs. (TC/TT) } \\
\hline & TT & $\mathbf{T C}$ & $\mathbf{C C}$ & TT & $\mathbf{T C}$ & $\mathbf{C C}$ & & \\
\hline \multicolumn{9}{|l|}{ Sex } \\
\hline Male & $309 / 447$ & $339 / 574$ & $95 / 170$ & 1.00 & $\begin{array}{c}0.82(0.68-1.00) \\
P: 0.054\end{array}$ & $\begin{array}{c}0.80(0.60-1.07) \\
P: 0.134\end{array}$ & $\begin{array}{c}0.86(0.71-1.04) \\
P: 0.109\end{array}$ & $\begin{array}{c}0.91(0.69-1.19) ; \\
P: 0.492\end{array}$ \\
\hline Female & $114 / 191$ & $147 / 234$ & $34 / 58$ & 1.00 & $\begin{array}{c}1.03(0.76-1.40) \\
P: 0.857\end{array}$ & $\begin{array}{c}0.99(0.61-1.61) \\
P: 0.970\end{array}$ & $\begin{array}{c}1.11(0.82-1.50) \\
P: 0.501\end{array}$ & $\begin{array}{c}1.01(0.64-1.60) ; \\
P: 0.956\end{array}$ \\
\hline \multicolumn{9}{|l|}{ Age } \\
\hline$<64$ & $190 / 288$ & $231 / 424$ & $59 / 111$ & 1.00 & $\begin{array}{c}0.78(0.61-0.99) \\
P: 0.040\end{array}$ & $\begin{array}{c}0.80(0.55-1.15) \\
P: 0.225\end{array}$ & $\begin{array}{c}0.83(0.66-1.05) \\
P: 0.128\end{array}$ & $\begin{array}{c}0.95(0.68-1.34) \\
P: 0.767\end{array}$ \\
\hline$\geq 64$ & $233 / 350$ & $255 / 384$ & $70 / 117$ & 1.00 & $\begin{array}{c}0.96(0.76-1.21) \\
P: 0.728\end{array}$ & $\begin{array}{c}0.89(0.63-1.25) \\
P: 0.502\end{array}$ & $\begin{array}{c}0.99(0.80-1.23) \\
P: 0.914\end{array}$ & $\begin{array}{c}0.93(0.68-1.28) \\
P: 0.651\end{array}$ \\
\hline \multicolumn{9}{|l|}{$\begin{array}{l}\text { Smoking } \\
\text { status }\end{array}$} \\
\hline Never & $299 / 499$ & $357 / 636$ & $95 / 186$ & 1.00 & $\begin{array}{c}0.88(0.73-1.06) \\
P: 0.182\end{array}$ & $\begin{array}{c}0.80(0.60-1.06) \\
P: 0.126\end{array}$ & $\begin{array}{c}0.92(0.77-1.11) \\
P: 0.380\end{array}$ & $\begin{array}{c}0.89(0.68-1.16) \\
P: 0.374\end{array}$ \\
\hline Ever & $124 / 140$ & $129 / 173$ & $34 / 43$ & 1.00 & $\begin{array}{c}0.83(0.59-1.16) \\
P: 0.270\end{array}$ & $\begin{array}{c}0.95(0.56-1.59) \\
P: 0.835\end{array}$ & $\begin{array}{c}0.87(0.63-1.19) \\
P: 0.378\end{array}$ & $\begin{array}{c}1.05(0.64-1.72) ; \\
P: 0.843\end{array}$ \\
\hline \multicolumn{9}{|c|}{$\begin{array}{l}\text { Alcohol } \\
\text { consumption }\end{array}$} \\
\hline Never & $357 / 564$ & $415 / 728$ & $113 / 213$ & 1.00 & $\begin{array}{c}0.86(0.72-1.03) \\
P: 0.092\end{array}$ & $\begin{array}{c}0.80(0.62-1.04) \\
P: 0.096\end{array}$ & $\begin{array}{c}0.90(0.76-1.06) \\
P: 0.214\end{array}$ & $\begin{array}{c}0.89(0.70-1.14) ; \\
P: 0.369\end{array}$ \\
\hline Ever & $66 / 74$ & $71 / 80$ & $16 / 15$ & 1.00 & $\begin{array}{c}0.93(0.57-1.49) \\
P: 0.750\end{array}$ & $\begin{array}{c}1.12(0.50-2.51) \\
P: 0.783\end{array}$ & $\begin{array}{c}0.97(0.61-1.54) \\
P: 0.907\end{array}$ & $\begin{array}{c}1.17(0.54-2.53) ; \\
P: 0.686\end{array}$ \\
\hline
\end{tabular}

a The genotyping was successful in 1063 (97.65\%) EGJA cases, and 1677 (99.82\%) controls for MTHFR rs9651118 T>C;

${ }^{\mathrm{b}}$ Adjusted for age, sex, smoking status and alcohol consumption (besides stratified factors accordingly) in a logistic regression model;

MTHFR rs4845882 AA genotype in male and $<64$ years subgroups. MTHFR rs9651118 $\mathrm{T}>\mathrm{C}$ is situated in intron 2 and possesses low LD with rs1801133 G>A $\left(r^{2}\right.$ $<0.30)$. Fuctional annotation by HapReg demonstrated that MTHFR rs9651118 $\mathrm{T}>\mathrm{C}$ coincides with MTHFR enhancers or promoters, which may correspond to the regions of open chromatin [28]. Several studies implicated that MTHFR rs9651118 C allele was associated with a reduced risk of lung cancer and prostate cancer [28, 29]. In addition, MTHFR rs9651118 C allele was associated with a decreased risk of breast cancer [30]. Our results suggested that MTHFR rs9651118 TC genotype may reduce EGJA susceptibility in $<64$ years subgroup, which were very similar to the findings of previous studies. In the future, these potential should be confirmed by functional studies.

In this case-control study, we constructed five MTHFR haplotypes to assess the potential inherited patterns of haplotype. We found that MTHFR $\mathrm{G}_{\mathrm{rs} 1801133} \mathrm{~T}_{\mathrm{r}}$ ${ }_{s 3753584} \mathrm{G}_{\mathrm{rs} 4845882} \mathrm{~A}_{\mathrm{rs} 4846048} \mathrm{C}_{\mathrm{rs} 9651118}, \mathrm{G}_{\mathrm{rs} 1801133} \mathrm{C}_{\mathrm{rs} 3753584} \mathrm{~A}_{\mathrm{rs} 4845882} \mathrm{~A}_{\mathrm{r}}$ ${ }_{\mathrm{s} 4846048} \mathrm{~T}_{\mathrm{rs} 9651118}$ and $\mathrm{G}_{\mathrm{rs} 1801133} \mathrm{~T}_{\mathrm{rs} 3753584} \mathrm{~A}_{\mathrm{rs} 4845882} \mathrm{G}_{\mathrm{rs} 4846048} \mathrm{~T}_{\mathrm{rs} 9651118}$ haplotypes significantly decreased the risk of EGJA. To the best of our knowledge, we first explore the relationship of haplotypes in MTHFR rs1801133 G>A, rs3753584 $\mathrm{T}>\mathrm{C}$, rs4845882 $\mathrm{G}>\mathrm{A}$, rs4846048 $\mathrm{A}>\mathrm{G}$ and $\mathrm{rs} 9651118$ $\mathrm{T}>\mathrm{C}$ polymorphisms with EGJA susceptibility. We also found that MTHFR rs $1801133 \mathrm{G}$ and rs4845882 A alleles might be protective factors for haplotype to EGJA.

However, several limitations in our study should be presented. First, for the controls were recruited from the local hospitals, the selection bias of the study population should not be ignored. Second, the data of plasma folate level were not available, which may affect the association between MTHFR SNPs and EGJA susceptibility. Thirdly, for lack of cancer stage, disease progression and overall survival data, we did not consider the influence of MTHFR 
Table 9: $M T H F R$ haplotype frequencies $(\%)$ in patients and controls and risk of esophagogastric junction adenocarcinom

\begin{tabular}{|c|c|c|c|c|c|c|}
\hline \multirow[t]{2}{*}{ Haplotypes } & \multicolumn{2}{|c|}{ Case $(n=2,126)$} & \multicolumn{2}{|c|}{ Control $(n=3,354)$} & \multirow[t]{2}{*}{ Crude OR (95\% CI) } & \multirow[t]{2}{*}{$P$} \\
\hline & $\mathbf{n}$ & $\%$ & $\mathbf{n}$ & $\%$ & & \\
\hline $\begin{array}{l}\mathrm{A}_{\mathrm{rs} 1801133} \mathrm{~T}_{\mathrm{rs} 3753584} \mathrm{G}_{\mathrm{rs} 48} \\
{ }_{45882} \mathrm{~A}_{\mathrm{rs} 4846048} \mathrm{~T}_{\mathrm{rs} 9651118}\end{array}$ & 814 & 39.36 & 1169 & 34.92 & 1.00 & \\
\hline $\begin{array}{l}\mathrm{G}_{\mathrm{rr} 1801133} \mathrm{~T}_{\mathrm{rs} 37535544} \mathrm{G}_{\mathrm{rs} 48} \\
{ }_{45882} \mathrm{~A}_{\mathrm{rs} 4846048} \mathrm{C}_{\mathrm{rs} 9651118}\end{array}$ & 708 & 34.24 & 1244 & 37.16 & $0.82(0.72-0.93)$ & 0.002 \\
\hline${ }_{45882}^{\mathrm{G}_{\mathrm{rs} 1801133}} \mathrm{~A}_{\mathrm{rr} 4846048} \mathrm{C}_{\mathrm{rs} 3753584} \mathrm{~A}_{\mathrm{rs} 4651118}$ & 171 & 8.27 & 335 & 10.01 & $0.69(0.56-0.85)$ & $<0.001$ \\
\hline $\begin{array}{l}\mathrm{G}_{\mathrm{rs} 1801133} \mathrm{~T}_{\mathrm{r} 33753584} \mathrm{~A}_{\mathrm{rs} 48} \\
{ }_{45882} \mathrm{G}_{\mathrm{rs} 4846048} \mathrm{~T}_{\mathrm{rs} 96511118}\end{array}$ & 173 & 8.37 & 309 & 9.23 & $0.80(0.65-0.99)$ & 0.038 \\
\hline $\begin{array}{l}\mathrm{G}_{\mathrm{rr} 1801133} \mathrm{~T}_{\mathrm{r} 33753584} \mathrm{G}_{\mathrm{rs} 48} \\
{ }_{45882} \mathrm{~A}_{\mathrm{rs} 4846048} \mathrm{~T}_{\mathrm{rs} 9651118}\end{array}$ & 130 & 6.29 & 206 & 6.15 & $0.91(0.72-1.15)$ & 0.416 \\
\hline Others & 72 & 3.48 & 85 & 2.54 & $1.22(0.88-1.69)$ & 0.239 \\
\hline
\end{tabular}

SNPs on progress and prognosis of EGJA. Last but not least, other environmental and genetic factors were not considered. Further studies are necessary to explore the effect of interactions between environment and gene factors on EGJA risk.

In conclusion, our study demonstrates that MTHFR rs $1801133 \mathrm{G}>\mathrm{A}$ may be associated with the increased risk of EGJA. Meanwhile, MTHFR rs3753584 T >C, rs4845882 $\mathrm{G}>\mathrm{A}$ and rs9651118 $\mathrm{T}>\mathrm{C}$ polymorphisms decrease the risk of EGJA in Eastern Chinese Han population. The further case-control studies are needed to confirm our findings.

\section{MATERIALS AND METHODS}

\section{Subjects}

Study conducted at the Affiliated Union Hospital of Fujian Medical University, Fujian Medical University Cancer Hospital and the Affiliated People's Hospital of Jiangsu University was approved by the Ethics Committee of Fujian Medical University (Fuzhou, China) and Jiangsu University (Zhenjiang, China). Subjects were enrolled from three hospitals in Eastern China. Our study involved 2,740 study participants, comprising 1,063 histopathologically confirmed sporadic EGJA patients and 1,677 healthy normal controls. Among them, 280 EGJA patients and 840 controls were enrolled from Fujian Medical University Union Hospital and Cancer Hospital of Fujian Medical University from January 2014 to May 2016. In addition, 783 EGJA patients and 837 controls were enrolled from the Affiliated People's Hospital of Jiangsu University between January 2008 and November 2016. All EJGA patients were Siewert type II. The control group involved normal individuals who visited these hospitals for health check. The healthy normal controls were unrelated to the EGJA patients and were cancer- free individuals. Data of demographic details and risk factors was obtained using a structured questionnaire. The definition of 'ever smokers' were subjects who smoked at least one cigarette per day over 1 year [11], and 'ever drinkers' were subjects who drank no less than three times a week for more than 6 months [11]. The corresponding data are listed in Table 1. The Ethical Committee of Fujian Medical University and Jiangsu University approved the study protocols (No. SQ2015-006-01 and No. 20150083, respectively).

\section{Selection of SNPs}

The MTHFR tagging SNPs (upstream and downstream of $M T H F R$ gene extending $5 \mathrm{~Kb}$, respectively) were selected from the database of $\mathrm{CHB}$ population using the HapMap Project (http://hapmap.ncbi.nlm.nih.gov/ index.html.en) and Haploview 4.2 software. The major criterion were: (a) MAF $\geq 0.05$ and call rate $\geq 95 \%$, (b) a HWE $P \geq 0.05$, (c) a pairwise linkage disequilibrium (LD) $r^{2}$ threshold of 0.8 between polymorphisms $\left(r^{2}>0.8\right)$ [11, 31, 32]. Finally, five MTHFR tagging SNPs (rs1801133 $\mathrm{G}>\mathrm{A}, \quad$ rs3753584 $\mathrm{T}>\mathrm{C}, \quad$ rs4845882 $\mathrm{G}>\mathrm{A}, \quad$ rs4846048 $\mathrm{A}>\mathrm{G}$ and rs9651118 $\mathrm{T}>\mathrm{C}$ ) were eligible and included in this case-control study to evaluate the effect of MTHFR polymorphisms with EGJA risk. The primary information of MTHFR tagging SNPs is presented in Table 2.

\section{DNA extraction and genotyping}

Each participant donated $2 \mathrm{ml}$ blood sample which was stored in an EDTA-anticoagulated tube. We use the Promega Genomic DNA Purification Kit (Promega, Madison, USA) to extract the genomic DNA. SNPscan ${ }^{\mathrm{TM}}$ genotyping assay (Genesky Biotechologies Inc., Shanghai, China) $[33,34]$ was harnessed to determine 
the genotyping of MTHFR rs $1801133 \mathrm{G}>\mathrm{A}$, rs3753584 $\mathrm{T}>\mathrm{C}, \mathrm{rs} 4845882 \mathrm{G}>\mathrm{A}, \mathrm{rs} 4846048 \mathrm{~A}>\mathrm{G}$ and $\mathrm{rs} 9651118$ $\mathrm{T}>\mathrm{C}$ polymorphisms. Briefly, 150ng DNA sample was denatured at $98^{\circ} \mathrm{C}$ for $5 \mathrm{~min}$. The ligation reaction was done in an ABI 2720 thermal cycler. We used a 48-plex fluorescence PCR reaction for each ligation product amplification. In an ABI 3730XL sequencer, PCR products were analyzed by capillary electrophoresis. The obtained raw data were conducted by GeneMapper 4.1 software (Applied Biosystems, USA). One hundred and ten DNA samples $(4 \%)$ were randomly selected to reanalyze the genotypes by different laboratory technicians and the reproducibility was $100 \%$.

\section{Statistical analysis}

Age of EGJA patients and controls was expressed as mean \pm standard deviation. And a Student's t-test was harnessed to assess the difference for age. The Chisquare test $\left(\chi^{2}\right)$ was used to compare age, sex, smoking, drinking and the genotypes distribution of MTHFR SNPs in patients and controls. We used multivariate logistic regression analysis to assess the risk of MTHFR rs1801133 G>A, rs3753584 $\mathrm{T}>\mathrm{C}$, rs4845882 $\mathrm{G}>\mathrm{A}$, rs4846048 $\mathrm{A}>\mathrm{G}$ and rs9651118 $\mathrm{T}>\mathrm{C}$ polymorphisms and considered the confounders such as sex, age, smoking and drinking status. The crude/adjusted ORs and 95\% CIs were calculated using the SAS software (Version 9.4; SAS Institute Inc., Cary, NC, USA). A $P<0.05$ (two sided) was considered as statistical significance. In this study, multiple comparisons were conducted by Bonferroni correction [35]. We used a SHESIS software (http:// analysis.bio-x.cn/myAnalysis.php) [17] to construct MTHFR haplotypes.

\section{ACKNOWLEDGMENTS}

We appreciate all subjects who participated in this study.We wish to thank Dr. Yan Liu (Genesky Biotechnologies Inc., Shanghai, China) for technical support.

\section{CONFLICTS OF INTEREST}

The authors have no potential conflicts of interest.

\section{GRANT SUPPORT}

This study was supported in part by Young and Middle-aged Talent Training Project of Health Development Planning Commission in Fujian Province (2016-ZQN-25 and 2014-ZQN-JC-11), Medical Innovation Project of Fujian Province (2014-CX-15 and 2014-CX-18), Nursery Garden Project of Fujian Medical
University (2015MP020), Science and Technology Project of Fujian Province (2060203), Natural Science Foundation of Universities and Colleges of Jiangsu Province (Grant No. 16KJB310002), Senior Talents Scientific Research Foundation of Jiangsu University (Grant No. 16JDG066) and Jiangsu Provincial Key R \& D Special Fund (BE2015666).

\section{REFERENCES}

1. Edgren G, Adami HO, Weiderpass E, Nyren O. A global assessment of the oesophageal adenocarcinoma epidemic. Gut. 2013; 62:1406-14. https://doi.org/10.1136/ gutjnl-2012-302412.

2. Dikken JL, Lemmens VE, Wouters MW, Wijnhoven BP, Siersema PD, Nieuwenhuijzen GA, van Sandick JW, Cats A, Verheij M, Coebergh JW, van de Velde CJ. Increased incidence and survival for oesophageal cancer but not for gastric cardia cancer in the Netherlands. Eur J Cancer. 2012; 48:1624-32. https://doi.org/10.1016/j.ejca.2012.01.009.

3. Jemal A, Siegel R, Xu J, Ward E. Cancer statistics, 2010. CA Cancer J Clin. 2010; 60:277-300. https://doi. org/10.3322/caac.20073.

4. Jemal A, Center MM, DeSantis C, Ward EM. Global patterns of cancer incidence and mortality rates and trends. Cancer Epidemiol Biomarkers Prev. 2010; 19:1893-907. https://doi.org/10.1158/1055-9965.EPI-10-0437.

5. de Jonge PJ, van Blankenstein M, Grady WM, Kuipers EJ. Barrett's oesophagus: epidemiology, cancer risk and implications for management. Gut. 2014; 63:191-202. https://doi.org/10.1136/gutjnl-2013-305490.

6. Lagergren J, Lagergren P. Recent developments in esophageal adenocarcinoma. CA Cancer J Clin. 2013; 63:232-48. https://doi.org/10.3322/caac.21185.

7. Hur C, Miller M, Kong CY, Dowling EC, Nattinger KJ, Dunn M, Feuer EJ. Trends in esophageal adenocarcinoma incidence and mortality. Cancer. 2013; 119:1149-58. https:// doi.org/10.1002/cncr.27834.

8. Giovannucci E, Rimm EB, Ascherio A, Stampfer MJ, Colditz GA, Willett WC. Alcohol, low-methionine--lowfolate diets, and risk of colon cancer in men. J Natl Cancer Inst. 1995; 87:265-73.

9. Pelucchi C, Talamini R, Negri E, Levi F, Conti E, Franceschi S, La Vecchia C. Folate intake and risk of oral and pharyngeal cancer. Ann Oncol. 2003; 14:1677-81.

10. Duthie SJ. Folic acid deficiency and cancer: Mechanisms of DNA instability. Br Med Bull. 1999; 55:578-92.

11. Tang W, Zhang S, Qiu H, Wang L, Sun B, Yin J, Gu H. Genetic variations in MTHFR and esophageal squamous cell carcinoma susceptibility in Chinese Han population. Med Oncol. 2014; 31:915. https://doi.org/10.1007/ s12032-014-0915-6.

12. Wang Y, Chen S, Kang M, Tang W, Gu H, Yin J, Huang Z. Genetic variations in MTHFR and gastric cardia 
adenocarcinoma susceptibility in the Chinese Han population. Int J Clin Exp Med. 2015; 8:18936-44.

13. Hajiesmaeil M, Tafvizi F, Sarmadi S. The effect of methylenetetrahydrofolate reductase polymorphisms on susceptibility to human papilloma virus infection and cervical cancer. Infect Genet Evol. 2016; 46:1-6. https:// doi.org/10.1016/j.meegid.2016.10.012.

14. Waseem M, Hussain SR, Kumar S, Serajuddin M, Mahdi F, Sonkar SK, Bansal C, Ahmad MK. Association of MTHFR (C677T) gene polymorphism with breast cancer in North India. Biomark Cancer. 2016; 8:111-7. https://doi. org/10.4137/BIC.S40446.

15. Song A, Zhao L, Li Y, Wu L, Li Y, Liu X, Lan S. Haplotypes of the MTHFR gene are associated with an increased risk of breast cancer in a Han Chinese population in Gansu province. IUBMB Life. 2016; 68:526-34. https://doi. org/10.1002/iub.1509.

16. Gutierrez-Alvarez O, Lares-Asseff I, Galaviz-Hernandez C, Reyes-Espinoza EA, Almanza-Reyes H, Sosa-Macias M, Chairez Hernandez I, Salas-Pacheco JM, Bailon-Soto CE. Involvement of MTHFR and TPMT genes in susceptibility to childhood acute lymphoblastic leukemia (ALL) in mexicans. Drug Metab Pers Ther. 2016; 31:41-6. https:// doi.org/10.1515/dmpt-2015-0036.

17. Shi YY, He L. SHEsis, a powerful software platform for analyses of linkage disequilibrium, haplotype construction, and genetic association at polymorphism loci. Cell Res. 2005; 15:97-8. https://doi.org/10.1038/sj.cr.7290272.

18. Blot WJ, Devesa SS, Kneller RW, Fraumeni JF Jr. Rising incidence of adenocarcinoma of the esophagus and gastric cardia. JAMA. 1991; 265:1287-9.

19. Hasegawa S, Yoshikawa T. Adenocarcinoma of the esophagogastric junction: incidence, characteristics, and treatment strategies. Gastric Cancer. 2010; 13:63-73. https://doi.org/10.1007/s10120-010-0555-2.

20. Kusano C, Gotoda T, Khor CJ, Katai H, Kato H, Taniguchi H, Shimoda T. Changing trends in the proportion of adenocarcinoma of the esophagogastric junction in a large tertiary referral center in Japan. J Gastroenterol Hepatol. 2008; 23:1662-5. https://doi. org/10.1111/j.1440-1746.2008.05572.x.

21. Zhou Y, Zhang Z, Zhang Z, Wu J, Ren D, Yan X, Wang Q, Wang Y, Wang H, Zhang J, Zhu X, Yang Y, Luo C, et al. A rising trend of gastric cardia cancer in Gansu Province of China. Cancer Lett. 2008; 269:18-25. https://doi. org/10.1016/j.canlet.2008.04.013.

22. Chai W, Zhang Z, Ni M, Geng P, Lian Z, Zhang G, Shi LL, Chen J. Genetic association between methylenetetrahydrofolate reductase gene polymorphism and risk of osteonecrosis of the femoral head. BioMed Res Int. 2015; 2015:196495. https://doi. org/10.1155/2015/196495.
23. Jain M, Pandey P, Tiwary NK, Jain S. MTHFR C677T polymorphism is associated with hyperlipidemia in women with polycystic ovary syndrome. J Hum Reprod Sci. 2012; 5:52-6. https://doi.org/10.4103/0974-1208.97802.

24. Shen $\mathrm{H}, \mathrm{Xu} \mathrm{Y}$, Zheng $\mathrm{Y}$, Qian $\mathrm{Y}, \mathrm{Yu} \mathrm{R}$, Qin $\mathrm{Y}$, Wang X, Spitz MR, Wei Q. Polymorphisms of 5,10-methylenetetrahydrofolate reductase and risk of gastric cancer in a chinese population: a case-control study. Int J Cancer. 2001; 95:332-6.

25. Miao X, Xing D, Tan W, Qi J, Lu W, Lin D. Susceptibility to gastric cardia adenocarcinoma and genetic polymorphisms in methylenetetrahydrofolate reductase in an at-risk chinese population. Cancer Epidemiol Biomarkers Prev. 2002; 11:1454-8.

26. Liu H, Jin G, Wang H, Wu W, Liu Y, Qian J, Fan W, Ma H, Miao R, Hu Z, Sun W, Wang Y, Jin L, et al. Association of polymorphisms in one-carbon metabolizing genes and lung cancer risk: a case-control study in chinese population. Lung Cancer. 2008; 61:21-9. https://doi.org/10.1016/j. lungcan.2007.12.001.

27. Shen H, Newmann AS, Hu Z, Zhang Z, Xu Y, Wang L, Hu X, Guo J, Wang X, Wei Q. Methylenetetrahydrofolate reductase polymorphisms/haplotypes and risk of gastric cancer: a case-control analysis in China. Oncol Rep. 2005; 13:355-60.

28. Lin VC, Lu TL, Yin HL, Yang SF, Lee YC, Liu CC, Huang CY, Yu CC, Chang TY, Huang SP, Bao BY. Prognostic relevance of methylenetetrahydrofolate reductase polymorphisms for prostate cancer. Int J Mol Sci. 2016; 17. https://doi.org/10.3390/ijms17121996.

29. Swartz MD, Peterson CB, Lupo PJ, Wu X, Forman MR, Spitz MR, Hernandez LM, Vannucci M, Shete S. Investigating multiple candidate genes and nutrients in the folate metabolism pathway to detect genetic and nutritional risk factors for lung cancer. PLoS One. 2013; 8:e53475. https://doi.org/10.1371/journal.pone.0053475.

30. Lu Q, Jiang K, Li Q, Ji YJ, Chen WL, Xue XH. Polymorphisms in the MTHFR gene are associated with breast cancer risk and prognosis in a chinese population. Tumour Biol. 2015; 36:3757-62. https://doi.org/10.1007/ s13277-014-3016-4.

31. Carlson CS, Eberle MA, Kruglyak L, Nickerson DA. Mapping complex disease loci in whole-genome association studies. Nature. 2004; 429:446-52. https://doi.org/10.1038/ nature 02623.

32. Tang W, Wang Y, Chen S, Lin J, Chen B, Yu S, Chen Y, $\mathrm{Gu} \mathrm{H}$, Kang M. Investigation of cytotoxic T-lymphocyte antigen 4 polymorphisms in gastric cardia adenocarcinoma. Scand J Immunol. 2016; 83:212-8. https://doi.org/10.1111/ sji.12409.

33. Zheng L, Yin J, Wang L, Wang X, Shi Y, Shao A, Tang W, Ding G, Liu C, Chen S, Gu H. Interleukin 1B rs16944 
$\mathrm{G}>\mathrm{A}$ polymorphism was associated with a decreased risk of esophageal cancer in a Chinese population. Clin Biochem. 2013; 46:1469-73. https://doi.org/10.1016/j. clinbiochem.2013.05.050.

34. Yin J, Wang L, Shi Y, Shao A, Tang W, Wang X, Ding G, Liu C, Chen S, Gu H. Interleukin 17A rs4711998 A>G polymorphism was associated with a decreased risk of esophageal cancer in a Chinese population. Dis Esophagus. 2014; 27:87-92. https://doi.org/10.1111/dote.12045.

35. Bland JM, Altman DG. Multiple significance tests: the bonferroni method. BMJ. 1995; 310:170. 